\title{
EDITORIAL
}

\section{¿Gadolinio sin restricciones?}

\section{Juan Pablo Cruz Quiroga ${ }^{*}$}

1. Radiólogo. Pontificia Universidad Católica de Chile. Santiago, Chile.

Se ha publicado la nueva declaración de consenso en conjunto del American College of Radiology y de la Natinoal Kidney Foundation en enero de este año1 sobre el uso de medios de contraste basados en gadolinio (MCBG) en pacientes con enfermedad renal crónica.

La publicación de múltiples casos de fibrosis nefrogénica sistémica (FNS) vinculados a los MCBG introdujo restricciones basadas en la función renal de los pacientes crónicos y, aún más, en pacientes con insuficiencia renal aguda y estados proinflamatorios en quienes existe una hiper activación de los macrófagos. Cabe destacar que la vasta mayoría de los casos fueron asociados al uso de MCBG con quelantes lineales, en que la estabilidad de la unión con el átomo de gadolinio es menor. Actualmente se ha hecho más frecuente el uso de los MCBG macrocíclicos en que la configuración del quelante permite una unión más estable, que fue motivado en gran medida por los reportes de depósito de gadolinio en el sistema nervioso central y médula ósea.

En esta declaración conjunta, se dividen los medios de contraste en tres clases distintas según su riesgo de FNS; los clase I, constituida por quelantes lineales, que tienen el mayor riesgo y mayor número de casos reportados; los clase II constituida por quelantes macrocíclicos y un lineal iónico (MultiHance ${ }^{\circledR}$, excreción hepato biliar parcial) en los cuales no hay casos confirmados que cumplan todos los criterios propuestos (determinados "unconfounded cases") que los vinculen a FNS; los clase III (Eovist ${ }^{\circledR}$, hepatobiliar específico) que se consideran de bajo riesgo, si bien los datos disponibles son escasos por el menor número de dosis administradas a nivel global.

¿Cuáles son los cambios o recomendaciones que se sugieren en esta nueva declaración? Como sabíamos de publicaciones previas, los riesgos de FNS se elevan en pacientes con insuficiencia renal crónica etapa 4/5 recibiendo MCBG lineales, en especial en dosis repetidas o dosis por sobre los rangos indicados. Se suma en esta declaración que (1) el riesgo de FNS con MCBG macrocíclicos es muy bajo con dosis estándar, incluso en pacientes con tasa de filtración glomerular $<30 \mathrm{~mL} / \mathrm{min} / 173 \mathrm{~m}^{2}$, por lo que el (2) tamizaje de función renal es opcional. (3) No existe profilaxis efectiva para la prevención de FNS. (4) En pacientes en diálisis, la administración de MCBG grupo II o III no debe alterar el esquema de diálisis, si bien se prefiere hemodiálisis por sobre peritoneo diálisis por su mayor efectividad en la remoción de los MCBG. (5) No se debe iniciar diálisis por administración de MCBG grupo II o III. (6) Si se requieren múltiples dosis de MCBG grupo II o III, no se debe retrasar la administración de una nueva dosis por temor a FNS, aunque de ser posible, se sugiere separar las dosis por >24 hrs. (7) Estas recomendaciones son aplicables en pacientes pediátricos, en pacientes con uso de drogas nefrotóxicas, quimioterapia o uso de contraste iodado previo.

Debemos mantener en cuenta eso si que, si bien se han liberalizado las restricciones, el uso de MCBG en resonancia magnética no puede ser considerado como de rutina y debe utilizarse siempre y cuando tenga el potencial de aportar información adicional sobre las secuencias no contrastadas.

\section{Referencias}

1. Weinreb JC, Rodby RA, Yee J, et al. Use of intravenous gadolinium-based contrast media in patients with kidney disease: Consensus statements from the American college of radiology and the national kidney foundation. Radiology. 2021; 298(1): 28-35. doi:10.1148/RADIOL.2020202903 